\title{
From chromosomes to genomics: the evolving trends
}

\author{
Amita Pal ${ }^{1} \cdot$ Rabindra Nath Chatterjee ${ }^{2}$
}

Published online: 6 December 2018

(c) Archana Sharma Foundation of Calcutta 2018

The journal Nucleus founded by Professors AK Sharma and Archana Sharma in 1958 is now in 61st year of its establishment. Placing the journal on strong footing, the founders left this material world in 2017 and 2008, respectively; with the expectation to sustain their mission by their well-wishers keeping pace with the progressive developments in scientific research. In order to cherish the memory of the founders of the journal the Editorial Board of the Nucleus desired to bring out this special issue.

There have been profound technical advances in chromosome researches since the foundation of this journal. Biology of the nucleus has made tremendous strides from microscope to molecules and from karyotyping of chromosome complements to Genomics. Therefore, for this issue a theme "From Chromosomes to Genomics: the evolving trends" has been suggested; keeping in view the historical perspective of chromosome techniques to whole genome sequencing - $\mathrm{a}$ 'VISION' which the founders of the journal continued to subscribe.

\section{Cytogenetics to cytogenomics and epigenetic regulation}

The articles included in this issue are invited from specialists covering an array of topics ranging from classical cytogenetics to cytogenomics and epigenetic regulation to structural organization of nucleome vis-a-vis evolving technical advances.

Amita Pal

amita@jcbose.ac.in

Rabindra Nath Chatterjee

chatterjee_rn@yahoo.co.in

1 Division of Plant Biology, Bose Institute, P 1/12 CIT Scheme VIIM, Kankurgachi, Kolkata 700054, India

2 Department of Zoology, University of Calcutta, Kolkata 700019, India
Beginning with the application of conventional cytological studies and tracing through cytogenomic approaches Gupta and Vashistha [https://doi.org/10.1007/s13237-0180243-x] provide lucid account of the progressive developments made on the cytogenetics of wheat, including development of aneuploid and deletion stocks, chromosome sorting and their implications in genome analysis, development of physical maps and whole genome sequencing. 'The Nucleus' is publishing this review at the milestone of 100 years since the discovery of ploidy in wheat. This is a useful article for wheat researchers to learn genetics and breeding of wheat.

Employing physical immuno-detection of DNA methylation in situ on chromosomes for elucidation of post-translational histone modification as epigenetic regulatory mark, Sharma et al. [https://doi.org/10.1007/s13237-018-0231-1] based on their studies on Dendrobium nobile, suggest that there is an enhancement in DNA methylation with ploidy change, indicating evolutionary divergence of histone code. However, the altered distribution of methylated histones does not affect the chromosomal distribution of histone phosphorylation. Epigenetic regulations of DNA methylation and post-translational histone modifications are inconsistent in the diploid and autopolyploid D. nobile.

\section{Nuclear protein, structural organization of nucleosome vis-a-vis evolving technical advances}

The degree of similarity amongst sequences of nuclear proteins involved in meiosis of different phylogenetic lineages was analysed [https://doi.org/10.1007/s13237-018-0253$8]$. These proteins diverged strongly between the different lineages. Whereas, the proteins involved in the chromatid strand exchange are more conserved; the structural proteins of synaptonemal complexes are less conserved. On the other hand, the nuclear architectural proteins help to wrap the supercoiled-DNA to maintain the chromatin in a very compact state to accommodate in the nucleus [https://doi. org/10.1007/s13237-018-0257-4]. The regulation of High 
Mobility Groups (HMGs) of proteins and their interactions with DNA modulate gene expression modifying the cellular phenotypes. These nuclear elements, namely HMGA, HMGN and HMG-Box (HMGB) play a multifaceted role in chromatin dynamics by facilitating interaction with nucleosomes, nucleosome-remodeling machines, transcription factors and histones, both in plants and animals.

Ponrathnam and Mishra [https://doi.org/10.1007/s1323 7-018-0242] described the modern approaches for deciphering the structural roles of nucleosome arrays in the genome of eukaryotes. They have emphasized that to understand functional relevance of nuclear protein one has to reveal spatial distribution of these proteins and RNA.

\section{Modulation of DNA damage repair to endure anticancerous therapy}

Topoisomerase 1 (Top1) and 2 (Top2) are common targets of anti-cancer drugs, which induce specific DNA lesions leading to cell death in tumour cells. An interesting article [https ://doi.org/10.1007/s13237-018-0248-5] dealt with modulation of natural ability of DNA damage repair of cells to withstand majority of the current anticancer therapies, such as, radiation therapy or harsh chemotherapies that create DNA lesions, leading to apoptosis and/or cell death.

\section{Genomics and its applications in plant biology}

High throughput next generation sequencing (NGS) has revolutionized the arena of genomics and is providing novel insights into various plant biological aspects. Extensive genome-wide transcriptome study mediated by NGS has transformed the study of stress biology at an unprecedented level. Transcriptomic modulation of glutathione (GSH) occurs under stress conditions in Arabidopsis thaliana [https://doi.org/10.1007/s13237-018-0256-5]. GSH serves as signalling molecule by stimulating various immuneresponsive genes and proteins.

Ubiquitination was initially perceived as a process of selective protein degradation. The review by Mandal et al. [https://doi.org/10.1007/s13237-018-0255-6] details about the ubiquitin-proteasome system (UPS) and its involvement in biotic and abiotic stress responses highlighting the application of genomics approaches in delineating the precise regulatory roles of UPS in modulating plant immune system.

\section{Role of non-coding RNA in functional genomics}

It has been revealed that besides protein-coding RNAs, large proportions of transcriptome include significant proportion of regulatory non protein-coding RNAs, which were earlier considered as 'transcriptional noises' or as 'junk RNA'. Subsequently, researchers have defied the myth of the longest member of the non-coding RNA family, long non-coding RNAs (lncRNAs), which act as stable and functional part of a genome. Important roles of IncRNAs reported so far in plants including stress responses are reviewed by Singh et al. [https://doi.org/10.1007/s13237-018-0249-49].

\section{Concluding remarks}

This thematic issue of Nucleus covers a wide spectrum of topics on cytogenetics and frontier aspects of genomics research in different organisms. It is hoped that the issue will provide researchers with new ideas and thoughts. We are grateful to all the authors for their dedication to contribute the articles, to UC Lavania, Editor-in-Chief, and M/s Springer Nature, for making available the facilities to edit the articles included in this issue along a theme dear to the Founder Editors of this journal as a fitting tribute to their memory. 